\title{
【光电工程／Optoelectronic Engineering】
}

\section{虚拟结构光照明双光子荧光显微成像}

\author{
郡永红，郑晓敏，汪 否，吴文帅，周 洁，陈嘉杰
}

深圳大学物理与光电工程学院, 光电器件与系统教育部/广东省重点实验室, 广东深圳 518060

摘 要: 结构光照明单光子荧光超分辨显微成像技术已经成为生命科学研究的重要手段. 为提高荧光 显微成像技术的分辨率与成像深度，提出虚拟结构光照明双光子荧光超分辨成像方法，建立虚拟结构光照 明双光子荧光超分辨成像理论模型, 并分别对 Lena 图像和 $100 \mathrm{~nm}$ 荧光珠进行模拟成像实验. 结果表明, 系统的空间分辨率提高了 1.95 倍. 这项超分辨成像技术既具有双光子荧光成像的光学切片能力与成像深度 大的优点, 还具有虚拟结构光照明超分辨成像的能力，对高散射的厚生物样本甚至活细胞动态超分辨成像 具有重要的潜在应用价值.

关键词: 光学工程; 荧光成像; 超分辨成像; 双光子成像; 结构光照明; 虚拟结构光照明

中图分类号：O4 文献标志码：A doi：10.3724/SP. J. 1249.2021.03221

\section{Two-photon virtual structured illumination microscopy}

\section{SHAO Yonghong, ZHENG Xiaomin, WANG Lei, WU Wenshuai, ZHOU Jie, and CHEN Jiajie}

\author{
College of Physics and Optoelectronic Engineering, Key Laboratory of Optoelectronic Devices and Systems of Ministry of
} Education and Guangdong Province, Shenzhen University, Shenzhen 518060, Guangdong Province, P. R. China

\begin{abstract}
One-photon structured illumination microscopy (SIM) is a powerful tool in the field of life science. To improve the resolution and imaging depth of fluorescence microscopy, we propose a novel super-resolution microscopy called two-photon virtual SIM ( $2 \mathrm{p}$-vSIM), and establish an imaging theory model for it. To verify the resolving ability of $2 p$-vSIM, we carry out the simulation experiments on the Lena image and $100 \mathrm{~nm}$ fluorescent beads. The results show that the imaging resolution is increased by a factor of 1.95 compared with conventional two-photon imaging technology. Our 2 p-vSIM has the advantages of optical sectioning and large imaging depth of two-photon microscopy as well as the super-resolution ability of virtual SIM, and enables super-resolution imaging of thick biological samples with high scattering and even dynamic super-resolution imaging of live cells.
\end{abstract}

Key words: optical engineering; fluorescence imaging; super-resolution microscopy; two-photon imaging; structured illumination; virtual structured illumination

苂光显微成像已广泛用于生物医学研究, 然而 由于光学衍射系统的限制, 可达到的最小分辨距离 约为 $200 \mathrm{~nm}$. 为提高光学系统分辨率, 出现了一系 列新型超分辨技术，如受激发射损耗 ( stimulated emission depletion, STED) 苂光显微技术、随机光学
重建显微技术 ( stochastic optical reconstruction microscopy, STORM) 和结构光照明显微成像技术 (structured illumination microscopy, SIM $)^{[1-5]}$. STED 技术将系统分辨率大幅提高至远高于衍射极限分辨 率水平 (几十 $\mathrm{nm}$ 或更高), 但所需的高功率受激辐

Received: 2020-11-02; Accepted: 2020-11-26

Foundation: National Natural Science Foundation of China (61775148, 61527827)

Corresponding author: Professor SHAO Yonghong. E-mail: shaoyh@ szu.edu.cn

Citation: SHAO Yonghong, ZHENG Xiaomin, WANG Lei, et al. Two-photon virtual structured illumination microscopy [J]. Journal of Shenzhen University Science and Engineering, 2021, 38(3): 221-226. (in Chinese) 
射耗尽激光对生物样品 (特别是活细胞) 损伤较大, 因此，不适于活细胞动态成像. STED 技术必须使 用特殊 STED 染料，这限制了样品的范围. STORM 技术的横向和轴向分辨率分别可达到高于 $20 \mathrm{~nm}$ 和 $50 \mathrm{~nm}$ 的水平，但重构一幅超分辨图像需要采集平 均数万张原始图像，限制了成像速度. STORM 技 术还需要具有开关效应的苂光探针, 对染料要求较 高，限制了其适用范围. SIM 技术可将成像分辨率 提高近 2 倍, 且不需要特殊染料和高功率激光, 光 学系统相对简单, 便于与其他系统兼容, 成像速度 快, 有利于活细胞成像 ${ }^{[6-7]}$.

在结构光超分辨成像技术中，采用光栅投影或 双光束干涉形式，在样品中产生强度正弦调制的结 构光图案，利用结构光与样本频谱的卷积作用，将 不可探测的高频信息转化为可探测的低频信息，通 过相移算法重构超分辨图像. 为了解这些已被混频 的高频信息，需获取不同相位下结构光照明产生的 苂光图案，如在线性结构光照明超分辨成像中，相 邻两幅结构光图案的相位差为 $2 \pi / 3$, 每个方向需 要采集 3 幅苂光图像, 3 个方向共需采集 9 幅苂光 图像，最终通过算法重构出超分辨图像，将分辨率 提高 2 倍. 然而，在这种结构光调制方式下，为减 小伪影, 获取高质量的超分辨图像, 需要精确控制 结构光图案的相移，大的相移误差会导致解频错误 而无法实现超分辨成像 ${ }^{[8-10]}$. 为了解决结构光相移 难的问题，研究提出虚拟结构光技术. 不同于传统 结构光成像技术利用硬件进行结构光调制，虚拟结 构光可以通过后期软件编程对苂光衍射图像进行结 构光调制，直接避免了相移误差，同样可以将分辨 率提高 2 倍, 并实现活体成像 ${ }^{[11-13]}$. 在视网膜形态 学研究中, 由于眼球运动不可避免, 导致很难获得 这类动态物体的高分辨率图像. 为满足眼球运动的 快速成像要求, 研究通过将虚拟结构光与行扫描成
像系统结合以提高成像速度，获得高分辨率的视网 膜活体成像 ${ }^{[14-16]}$.

对于具有一定厚度的易散射样品，单光子过程 的激发深度被限制在样品表面以下几十 $\mu \mathrm{m}$; 随着 成像深度的增加，信噪比下降导致分辨率的显著下 降，限制了虚拟结构光技术的超分辨能力. 多光子 效应荧光成像具有穿透深度大、光损伤小和光漂白 小等优势, 已成为生物医学研究的重要手段 ${ }^{[17-18]}$. 然而, 由于双光子过程的非线性响应, 导致激发点 扩展函数与发射点扩展函数不能近似相等，因此, 线性结构光公式不能直接用于双光子虚拟结构光.

为同时提高成像深度与系统分辨率，本研究将 多光子效应与虚拟结构光技术相结合，提出虚拟结 构光照明双光子苂光超分辨成像方法，推导成像理 论公式，模拟成像实验. 结果证明，该方法能够将 分辨率提高 1.95 倍, 可用于高散射厚样本的超分 辨成像.

\section{1 虚拟结构光照明双光子荧光成像原理}

\section{1 光学成像系统}

虚拟结构光照明双光子苂光成像系统采用点扫 描激发与面探测相结合光路, 如图 1. 飞秒激光器 产生近红外飞秒脉冲激光, 为避免高功率激光反射 回激光谐振器干扰锁模, 需在激光器后放置光隔离 器. 激光经 $x-y$ 轴振镜扫描后到达管镜, 激发滤光 片用于过滤激发光以外的干扰光，再以高数值孔径 物镜聚焦至样品, 通过双光子过程激发样品产生苂 光，苂光经由物镜后进人二向色镜反射至发射滤光 片，最后被面探测器采集. 由于双光子效应，仅焦 点处的样本可被激发出苂光，从而减少了焦平面外 的背景信号, 提高了光学切片能力.

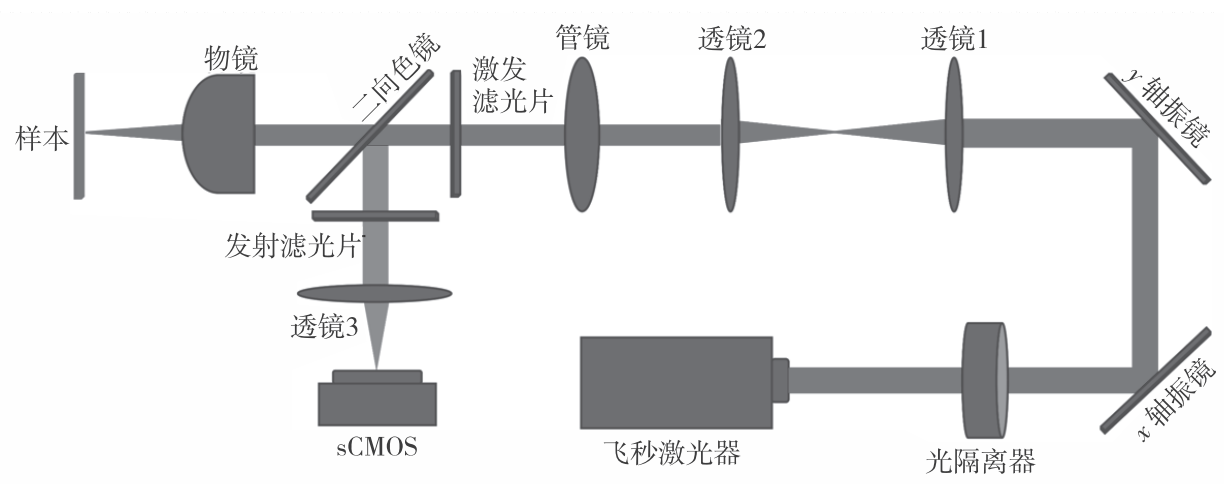

图 1 虚拟结构光照明双光子荧光成像系统光路示意图

Fig. 1 The scheme of two-photon virtual structured illumination microscopy 


\section{2 虚拟结构光照明双光子荧光成像理论}

通过点扫描将样本逐点激发. 由于存在物镜衍 射, 每个激发点的激发光通过物镜后, 在焦面处被 衍射成为一个艾里斑, 因此, 除物镜焦点处的样本 被激发产生苂光外, 焦点附近的样本也被激发. 在 双光子效应中, 苂光强度与激发光强度的平方成正 比, 因此, 产生的苂光光强与激发点扩散函数的平 方成正比．每个激发点激发出的苂光通过物镜被面 探测器 ( scientific complementary metal-oxide semiconductor, sCMOS) 成像记录, 探测器不仅记录到焦点 处的样本信息, 也记录到焦点外艾里斑内的样本信 息, 这为后期的超分辨提供可能. 通过逐点扫描和 逐幅记录, 可以获得一系列点样本的衍射斑图. 以 上成像过程可表示为

$$
\begin{aligned}
& \operatorname{imag}\left(\boldsymbol{r}, \boldsymbol{r}_{\mathrm{s}}, \boldsymbol{r}_{\mathrm{d}}\right)^{(n)}= \\
& \quad h_{\mathrm{ex}}^{2}\left(\boldsymbol{r}_{\mathrm{s}}-\boldsymbol{r}\right) s\left(\boldsymbol{r}_{\mathrm{s}}\right) h_{\mathrm{em}}\left(\boldsymbol{r}_{\mathrm{d}}-\boldsymbol{r}_{\mathrm{s}}\right)
\end{aligned}
$$

其中, $\operatorname{imag}\left(r, r_{\mathrm{s}}, \boldsymbol{r}_{\mathrm{d}}\right)^{(n)}$ 表示采集到的第 $n$ 个衍射斑 图像; $r$ 为扫描位置坐标; $r_{\mathrm{s}}$ 为样本面坐标; $r_{\mathrm{d}}$ 为成 像面坐标; $h_{\mathrm{ex}}(\boldsymbol{r})$ 和 $h_{\mathrm{em}}(\boldsymbol{r})$ 分别表示激发点扩散函 数和发射点扩散函数, 均由光学系统决定, 且与光 波长有关; $s(\boldsymbol{r})$ 表示样本菼光团分布.

为讨论方便, 以下以扫描点坐标的横坐标 $x$ 分 量 $r_{x} 、 r_{\mathrm{s} x}$ 及 $r_{\mathrm{d} x}$ 代替 $\boldsymbol{r} 、 \boldsymbol{r}_{\mathrm{s}}$ 及 $\boldsymbol{r}_{\mathrm{d}}$ 进行推导. 将每张衍射 斑图都乘以相同的正弦条纹进行调制, 然后将所有 像素相加后赋值给焦点位置, 得到样本的总衍射图 像，可表示为

$$
\begin{aligned}
& i\left(r_{x}\right)=\iint \operatorname{imag}\left(r_{x}, r_{\mathrm{s} x}, r_{\mathrm{d} x}\right)^{(n)} p\left(r_{\mathrm{d} x}\right) \mathrm{d} r_{\mathrm{s} x} \mathrm{~d} r_{\mathrm{d} x} \\
& p\left(r_{\mathrm{d} x}\right)=\frac{1}{2}\left[1+\cos \left(2 \pi k_{0} r_{\mathrm{d} x}+\varphi\right)\right]
\end{aligned}
$$

其中, $i\left(r_{x}\right)$ 为结构光调制混频后的总衍射图像; $p\left(r_{\mathrm{d} x}\right)$ 为调制函数; $k_{0}$ 为空间频率; $\varphi$ 为相位.

将 $p\left(r_{\mathrm{d} x}\right)$ 代人 $i\left(r_{x}\right)$ 得

$$
\begin{gathered}
i\left(r_{x}\right)=\iint h_{\mathrm{ex}}^{2}\left(r_{\mathrm{sx}}-r_{x}\right) s\left(r_{\mathrm{sx}}\right) h_{\mathrm{em}}\left(r_{\mathrm{d} x}-r_{\mathrm{sx}}\right) p\left(r_{\mathrm{d} x}\right) \mathrm{d} r_{\mathrm{sx}} \mathrm{d} r_{\mathrm{d} x}= \\
\int h_{\mathrm{ex}}^{2}\left(r_{\mathrm{s} x}-r_{x}\right) s\left(r_{\mathrm{s} x}\right)\left[h_{\mathrm{em}}\left(r_{\mathrm{s} x}\right) * p\left(r_{\mathrm{sx}}\right)\right] \mathrm{d} r_{\mathrm{s} x}= \\
\left\{s\left(r_{x}\right)\left[h_{\mathrm{em}}\left(r_{x}\right) * p\left(r_{x}\right)\right]\right\} * h_{\mathrm{ex}}^{2}\left(r_{x}\right)
\end{gathered}
$$

其中, $*$ 是卷积运算. 在传统结构光成像中, 调制 函数的空间频率受系统激发点扩散函数的限制, 使 结构光超分辨技术最多可使分辨率提高 2 倍. 由式 (4) 可见，在双光子虚拟结构光中，由于积分运算， 激发点扩散函数与发射点扩散函数位置颠倒, 此时 限制调制函数空间频率的是发射点扩散函数. 在双 光子效应中, 激发点扩散函数的平方与发射点扩散 函数差异不大, 可忽略其差异, 因此, 最终成像效 果与传统结构光成像技术相同.

以下对该混频图像进行解频并重建超分辨图 像. 对式(4)进行傅里叶变换得

$$
\begin{aligned}
I(k)= & \left\{\left[P(k) \operatorname{OTF}_{\text {em }}(k)\right] * S(k)\right\} \times \\
& {\left[\operatorname{OTF}_{\text {ex }}(k) * \mathrm{OTF}_{\text {ex }}(k)\right] } \\
P(k)= & \delta(k)+\frac{1}{2} \delta\left(k-k_{0}\right) \mathrm{e}^{\mathrm{i} \varphi}+ \\
& \frac{1}{2} \delta\left(k+k_{0}\right) \mathrm{e}^{-\mathrm{i} \varphi}
\end{aligned}
$$

其中, $k$ 为频域坐标; $P(k) 、 S(k) 、 \mathrm{OTF}_{\text {ex }}(k)$ 和 $\mathrm{OTF}_{\mathrm{em}}(k)$ 分别为 $p\left(r_{x}\right) 、 s\left(r_{x}\right) 、 h_{\mathrm{ex}}\left(r_{x}\right)$ 和 $h_{\mathrm{em}}\left(r_{x}\right)$ 的傅里叶变换. 将式 (6) 代人式 (5) 得

$$
\begin{aligned}
I(k)= & {\left[S(k)+\frac{1}{2} S\left(k-k_{0}\right) \mathrm{e}^{\mathrm{i} \varphi} \mathrm{OTF}_{\mathrm{em}}\left(k_{0}\right)+\right.} \\
& \left.\frac{1}{2} S\left(k+k_{0}\right) \mathrm{e}^{-\mathrm{i} \varphi} \mathrm{OTF}_{\mathrm{em}}\left(-k_{0}\right)\right] \times \\
& {\left[\operatorname{OTF}_{\mathrm{ex}}(k) * \mathrm{OTF}_{\mathrm{ex}}(k)\right] }
\end{aligned}
$$

为了能够解出 $S(k)\left[\mathrm{OTF}_{\mathrm{ex}}(k) * \mathrm{OTF}_{\mathrm{ex}}(k)\right]$ 、 $\mathrm{OTF}_{\mathrm{em}}\left(k_{0}\right) S\left(k-k_{0}\right)\left[\mathrm{OTF}_{\mathrm{ex}}(k) * \mathrm{OTF}_{\mathrm{ex}}(k)\right]$ 和 $\mathrm{OTF}_{\mathrm{em}}\left(-k_{0}\right) S\left(k+k_{0}\right)\left[\mathrm{OTF}_{\mathrm{ex}}(k) * \mathrm{OTF}_{\mathrm{ex}}(k)\right]$ 分 量, 需要建立 3 个相位方程, 即

$$
\left[\begin{array}{c}
I_{1}(k) \\
I_{2}(k) \\
I_{3}(k)
\end{array}\right]=\left[\begin{array}{ccc}
1 & \frac{1}{2} \mathrm{e}^{\mathrm{i} \varphi_{1}} & \frac{1}{2} \mathrm{e}^{-\mathrm{i} \varphi_{1}} \\
1 & \frac{1}{2} \mathrm{e}^{\mathrm{i} \varphi_{2}} & \frac{1}{2} \mathrm{e}^{-\mathrm{i} \varphi_{2}} \\
1 & \frac{1}{2} \mathrm{e}^{\mathrm{i} \varphi_{3}} & \frac{1}{2} \mathrm{e}^{-\mathrm{i} \varphi_{3}}
\end{array}\right]\left[\begin{array}{c}
S(k)\left[\operatorname{OTF}_{\text {ex }}(k) * \operatorname{OTF}_{\text {ex }}(k)\right] \\
\operatorname{OTF}_{\text {em }}\left(k_{0}\right) S\left(k-k_{0}\right)\left[\operatorname{OTF}_{\text {ex }}(k) * \mathrm{OTF}_{\text {ex }}(k)\right] \\
\operatorname{OTF}_{\text {em }}\left(-k_{0}\right) S\left(k+k_{0}\right)\left[\operatorname{OTF}_{\text {ex }}(k) * \operatorname{OTF}_{\text {ex }}(k)\right]
\end{array}\right]
$$

对应的解频公式为

$$
\left[\begin{array}{c}
S(k)\left[\mathrm{OTF}_{\text {ex }}(k) * \mathrm{OTF}_{\text {ex }}(k)\right] \\
\operatorname{OTF}_{\text {em }}\left(k_{0}\right) S\left(k-k_{0}\right)\left[\operatorname{OTF}_{\text {ex }}(k) * \operatorname{OTF}_{\text {ex }}(k)\right] \\
\operatorname{OTF}_{\text {em }}\left(-k_{0}\right) S\left(k+k_{0}\right)\left[\operatorname{OTF}_{\text {ex }}(k) * \operatorname{OTF}_{\text {ex }}(k)\right]
\end{array}\right]=\left[\begin{array}{lll}
1 & \frac{1}{2} \mathrm{e}^{\mathrm{i} \varphi_{1}} & \frac{1}{2} \mathrm{e}^{-\mathrm{i} \varphi_{1}} \\
1 & \frac{1}{2} \mathrm{e}^{\mathrm{i} \varphi_{2}} & \frac{1}{2} \mathrm{e}^{-\mathrm{i} \varphi_{2}} \\
1 & \frac{1}{2} \mathrm{e}^{\mathrm{i} \varphi_{3}} & \frac{1}{2} \mathrm{e}^{-\mathrm{i} \varphi_{3}}
\end{array}\right]^{-1}\left[\begin{array}{c}
I_{1}(k) \\
I_{2}(k) \\
I_{3}(k)
\end{array}\right]
$$


由式(9) 可见, 解频需要 3 步相移, 可以通过 软件编程获得 3 幅相移 (如 $\varphi_{1}=0, \varphi_{2}=\frac{2 \pi}{3}$, $\left.\varphi_{3}=\frac{4 \pi}{3}\right)$ 的混频图像. 为实现均匀频域展宽, 至 少需要 3 个方向 $\left(\right.$ 如 $0^{\circ} 、 60^{\circ}$ 和 $120^{\circ}$ ) 的结构光图 案调制. 由于调制函数由软件编程产生, 因此, 可 获得相位的精确赋值, 避免了传统结构光技术中的 相移误差, 从而能准确解频重建超分辨图像.

\section{2 实验}

\subsection{Lena 图像模拟实验}

为了定性验证双光子虚拟结构光超分辨成像效 果, 对 Lena 图像进行模拟成像. 设置单位像素长 度为 $40 \mathrm{~nm}$, 激发光波长为 $800 \mathrm{~nm}$, 样品发射光波
长为 $560 \mathrm{~nm}$, 数值孔径 (numerical aperture, NA) 为 1. 4 , 则由瑞利判据可得到系统的最小分辨距离为

$$
\frac{0.61 \lambda}{\mathrm{NA}}=244 \mathrm{~nm}
$$

图 2 为传统双光子与虚拟结构光照明双光子的 模拟成像 Lena 图片. 对图 2 (a) 的无衍射样本图像 以双光子点扫描形式进行模拟成像，可得如图 2 (b) 的传统双光子衍射图像; 再对所得双光子衍射 斑图像集按照虚拟结构光照明双光子苂光超分辨成 像方法进行混频调制，可以得到混频图像. 最后通 过对该混频图像解频并重建, 得到更高分辨率的超 分辨图像, 如图 2 (c). 对比图 2 矩形框内的高频 条纹图像可见, 图 2 (c) 可以分辨这些条纹, 而图 2 (b)不能分辨，证明虚拟结构光照明双光子荧光 成像具有超分辨的成像能力.

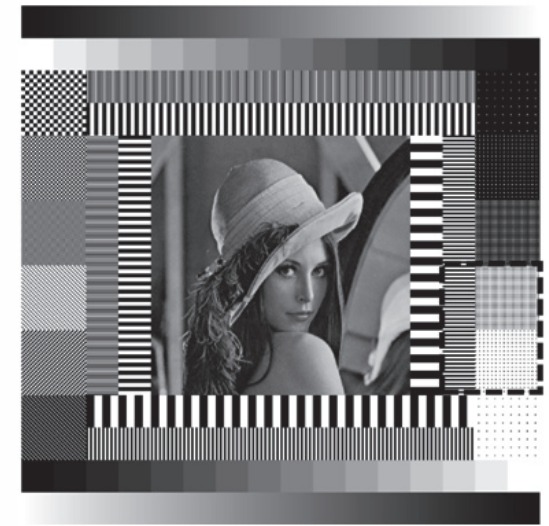

(a) 无衍射Lena图像

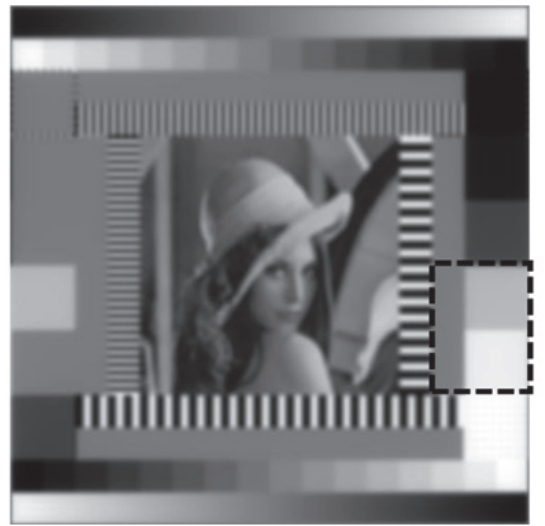

(b) 传统双光子衍射

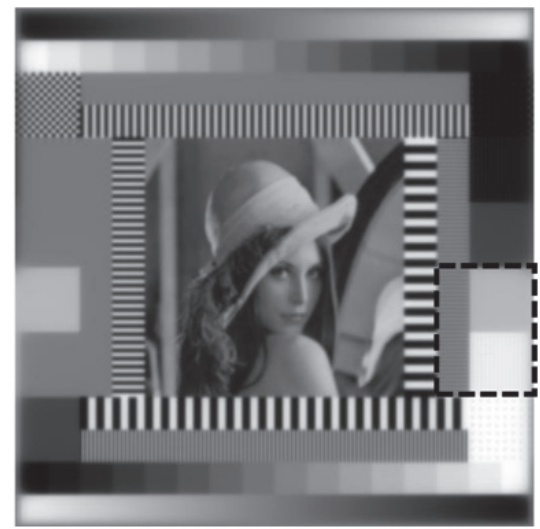

（c）虚拟结构光照明双光子苂光成像重建图像

图 2 传统双光子与虚拟结构光照明双光子模拟成像 Lena 图片

Fig. 2 Simulated imaging of Lena picture for conventional two-photon microscopy and two-photon virtual SIM

\section{2 荧光珠实验}

为定量分析虚拟结构光照明双光子苂光超分辨 成像能力, 通过模拟实验对 $100 \mathrm{~nm}$ 荧光珠进行传 统双光子与虚拟结构光照明双光子成像对比研究. 成像条件与 Lena 图像实验相同. 模拟得到的传统 双光子图像如图 3 (a). 可见，无法分辨图 3 (a) 中 实线段位置的苂光珠. 以点扫描形式对样本进行虚 拟结构光照明双光子苂光成像，即激发光斑每移动 1 个位置，记录 1 幅艾里斑图像，得到一系列衍射 斑图；根据虚拟结构光照明双光子苂光成像方法， 对衍射斑图逐幅乘以相同的正弦条纹并积分进行混
频调制；最后通过结构光重建算法恢复出超分辨图 像，如图 3 (b). 可见，虚拟结构光照明双光子苂 光成像能够将图 3 (a) 中实线段位置的荧光珠分开. 为了定量描述分辨率能力, 分别选择了 1 个和 3 个 相近的荧光珠，沿图 3(a) 或图 3(b) 中虚线段或实 线段位置获取强度分布，如图 $3(\mathrm{c})$ 和图 $3(\mathrm{~d})$. 由 图 3 (c) 可见, 单个苂光珠的半高宽分别为 $235 \mathrm{~nm}$ 和 $120 \mathrm{~nm}$ ，虚拟结构光照明双光子荧光成像将分辨 率提高了 1.95 倍; 为展示频谱展宽效果，绘制相 应频谱分布图，如图 3 (e) 和图 3 (f), 虚拟结构光 照明双光子苂光成像实现了频谱均匀拓宽. 


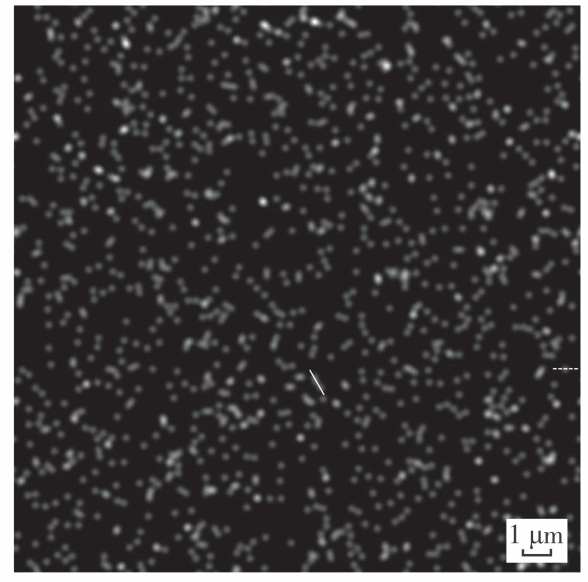

(a) 传统双光子模拟成像 $100 \mathrm{~nm}$ 苂光珠

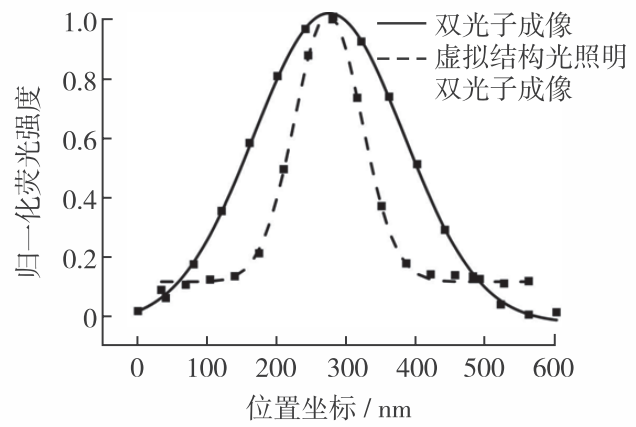

(c) 图 (a) 和 (b) 中虚线段处的苂光珠强度曲线

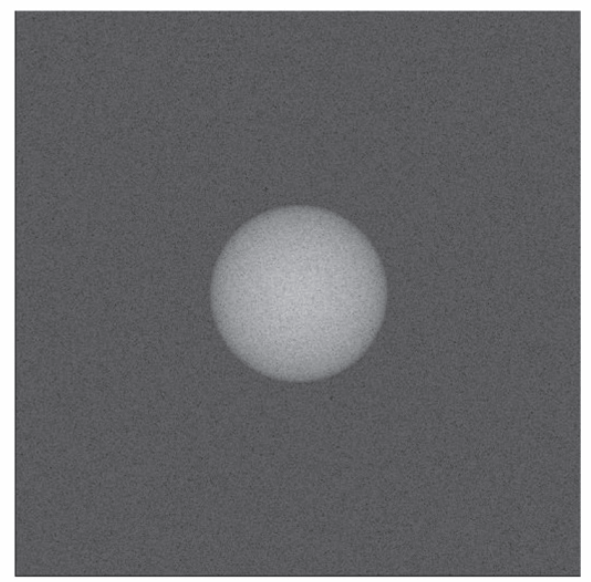

(e) 图 (a) 的频谱图

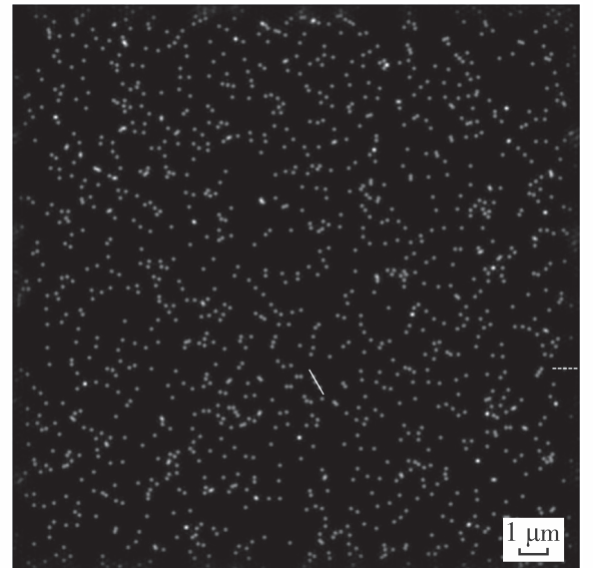

（b）虚拟结构光照明双光子模拟成像 $100 \mathrm{~nm}$ 苂光珠

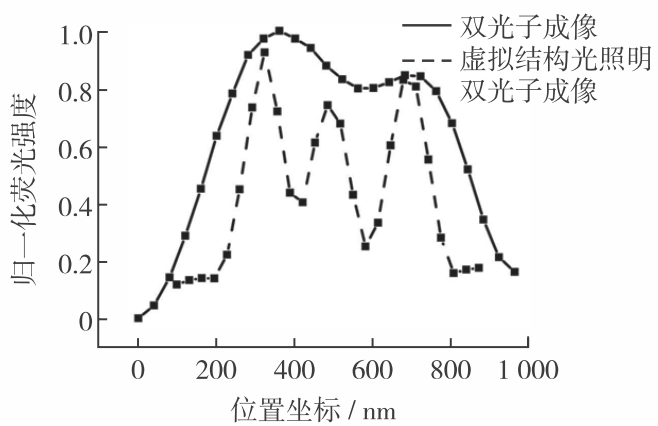

(d) 图 (a) 和 (b) 中实线段处的苂光珠强度曲线

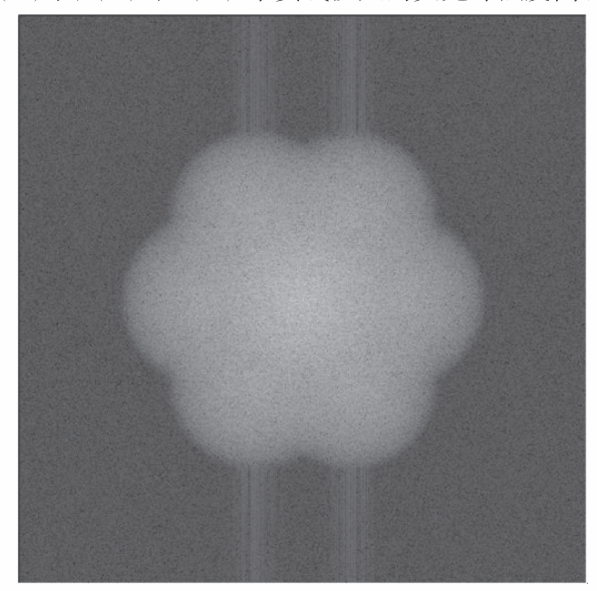

(f) 图 (b) 的频谱图

图 3 传统双光子与虚拟结构光照明双光子模拟成像 $100 \mathbf{n m}$ 荧光珠

Fig. 3 Comparison of resolution capability between conventional two-photon microscopy and two-photon virtual SIM by simulated imaging of $100 \mathrm{~nm}$ fluorescent beads

\section{结 语}

针对厚组织的高分辨率成像需求, 提出虚拟结 构光照明双光子苂光成像技术, 设计该方法的光学 系统结构, 推导成像原理, 建立虚拟结构光照明双 光子苂光超分辨图像的重构算法, 并通过模拟验证 该方法有效. 结果表明, 虚拟结构光照明双光子菼
光成像能够将传统双光子成像分辨率提高 1.95 倍, 对厚样本的高分辨率成像具有重要意义.

尽管虚拟结构光照明双光子苂光成像可以避免 精确控制相移的问题, 但存在采集幅数多, 成像速 度慢的缺点. 多点并行技术已经成为提高成像速度 的有效手段，因此，将来结合线扫描或多焦点扫描 方案, 可以提高成像速度, 并降低数据量, 有利于 虚拟结构光照明双光子苂光超分辨图像重构算法的 
数据处理. 在生物成像应用方面, 虚拟结构光照明 双光子苂光成像具有成像深度大的优势，适于厚度 较大生物样品成像研究. 通过结合线扫描或多焦点 扫描技术，有望实现活细胞动态超分辨成像. 虚拟 结构光照明双光子荧光成像技术在生物医学研究方 面具有良好应用前景.

基金项目: 国家自然科学基金资助项目 $(61775148,61527827)$

作者简介: 邵永红 (1972-), 深圳大学教授、博士生导师. 研究 方向: 生物医学光子学显微成像与传感. E-mail: shaoyh@ szu.edu.cn

引文: 邵永红, 郑晓敏, 汪 否, 等. 虚拟结构光照明双光子 苂光显微成像 $[\mathrm{J}]$. 深圳大学学报理工版, 2021，38 (3) : 221-226

\section{参考文献 / References:}

[ 1 ] KLAR T A, HELL S W. Subdiffraction resolution in far-field fluorescence microscopy $[\mathrm{J}]$. Optics Letters, 1999, 24(14) : 954-956.

[ 2 ] KLEIN T, PROPPERT S, SAUER M. Eight years of singlemolecule localization microscopy $[\mathrm{J}]$. Histochemistry and Cell Biology, 2014, 141(6) : 561-575.

[ 3 ] GUSTAFSSON M G L. Surpassing the lateral resolution limit by a factor of two using structured illumination microscopy [J]. Journal of Microscopy, 2000, 198(2) : 82-87.

[4] 林丹樱, 屈军乐. 超分辨成像及超分辨关联显微技术 研究进展 $[\mathrm{J}]$. 物理学报, 2017, 66(14)：271-293.

LIN Danying, QU Junle. Recent progress on super-resolution imaging and correlative super-resolution microscopy [J]. Acta Physica Sinica, 2017, 66(14) : 271-293. (in Chinese)

[ 5 ] 张崇磊, 辛自强, 闵长俊, 等. 表面等离激元结构光照 明显微成像技术研究进展 $[\mathrm{J}]$. 物理学报, 2017, 66 (14) : 239-255.

ZHANG Chonglei, XIN Ziqiang, MIN Changjun, et al. Research progress of plasmonic structure illumination microscopy [J] Acta Physica Sinica, 2017, 66 (14): 239-255. (in Chinese)

[ 6 ] LI Dong, SHAO Lin, CHEN Bichang, et al. Extendedresolution structured illumination imaging of endocytic and cytoskeletal dynamics $[\mathrm{J}]$. Science, 2015, 349(6251): aab3500.

[ 7 ] MARKWIRTH A, LACHETTA M, MONKEMOLLER V, et al. Video-rate multi-color structured illumination microscopy with simultaneous real-time reconstruction [J]. Nature Communications, 2019, 10 (1) : 4315.

[ 8 ] LAL A, SHAN Chunyan, XI Peng. Structured illumination microscopy image reconstruction algorithm $[\mathrm{J}]$. IEEE Journal of Selected Topics in Quantum Electronics, 2016, $22(4): 6803414$

[ 9 ] SHROFF S A, FIENUP J R, WILLIAMS D R. Phase-shift estimation in sinusoidally illuminated images for lateral superresolution $[\mathrm{J}]$. Journal of the Optical Society of America A, 2009, 26(2) : 413-424.

[10] 赵天宇, 周 兴, 但 旦, 等. 结构光照明显微中的偏 振控制 $[\mathrm{J}]$. 物理学报, 2017, 66(14): 288-299.

ZHAO Tianyu, ZHOU Xing, DAN Dan, et al. Polarization control methods in structured illumination microscopy [J]. Acta Physica Sinica, 2017, 66 (14) : 288-299. (in Chinese)

[11] KUANG Cuifang, MA Ye, ZHOU Renjie, et al. Virtual $k$-space modulation optical microscopy $[\mathrm{J}]$. Physical Review Letters, 2016, 117(2) : 028102.

[12] CAO Ruizhi, KUANG Cuifang, LIU Yong, et al. Superresolution via saturated virtual modulation microscopy [J]. Optics Express, 2017, 25(26) : 32364-32379.

[13] LU Rongwen, WANG Benquan, ZHANG Qiuxiang, et al. Super-resolution scanning laser microscopy through virtually structured detection $[\mathrm{J}]$. Biomedical Optics Express, $2013,4(9)$ : 1673-1682.

[14] ZHI Ya'nan, LU Rongwen, WANG Benquan, et al. Rapid super-resolution line-scanning microscopy through virtually structured detection $[\mathrm{J}]$. Optics Letters, 2015, 40(8): $1683-1686$

[15] LIU Changgeng, ZHI Ya'nan, WANG Benquan, et al. In vivo super-resolution retinal imaging through virtually structured detection $[\mathrm{J}]$. Journal of Biomedical Optics, 2016, 21(12) : 120502.

[16］高兴宇, 萧泽新, 伍世荣. 线结构光共焦显微成像技术 的实验研究 $[J]$. 光学技术, 2006,32 (增刊 1 )：173175 .

GAO Xingyu, XlAO Zexin, WU Shirong. The experiment research of line constructive laser confocal microscope technique $[\mathrm{J}]$. Optical Technique, 2006, 32(Suppl. 1) : 173-175. (in Chinese)

[17] KÖNIG K. Multiphoton microscopy in life sciences [ J]. Journal of Microscopy, 2010, 200( Pt 2) : 83-104.

[18 ] ISOBE K, TAKEDA T, MOCHIZUKI K, et al. Enhancement of lateral resolution and optical sectioning capability of two-photon fluorescence microscopy by combining temporal-focusing with structured illumination $[\mathrm{J}]$. Biomedical Optics Express, 2013, 4(11) : 2396-2410.

【中文责编：方 圆; 英文责编：溯 心】 